\title{
Changes in Cephalometric Variables after Orthognathic Surgery and Their Relationship to Patients' Quality of Life and Satisfaction
}

\author{
Tahereh Baherimoghaddam ${ }^{1}$, Morteza Oshagh ${ }^{2}$, Navid Naseri ${ }^{3}$, Nariman Iraji Nasrbadi ${ }^{3}$, \\ Sepideh Torkan ${ }^{4}$
}

${ }^{1}$ Department of Orthodontics, School of Dentistry, Yasuj University of Medical Sciences, Yasuj, Iran.

${ }^{2}$ Private practice, Tehran, Iran.

${ }^{3}$ Department of Orthodontics, School of Dentistry, Islamic Azad University, Tehran, Iran.

${ }^{4}$ Department of Orthodontics, School of Dentistry, Shiraz University of Medical Sciences, Shiraz, Iran.

\author{
Corresponding Author: \\ Tahereh Baherimoghadam \\ Faculty of Dentistry, Department of Orthodontics \\ Yasuj University of Medical Sciences \\ Kohkiluyeh Va Boyer Ahmad 7591994799, Yasuj \\ Iran \\ Phone: +989124190940 \\ Fax: +987116280801 \\ E-mail: t baheri@yahoo.com
}

\begin{abstract}
Objectives: This study was designed to assess the relationship between postsurgical cephalometric changes and quality of life and satisfaction after orthognathic surgery.

Material and Methods: Sample size consisted of 30 patients with class III and 25 patients with class II malocclusion treated with bilateral sagittal split osteotomy and Le Fort I osteotomy. Profile convexity, lower facial height proportion, lip position and length, nose, and chin position were measured on pre and post-treatment cephalograms. To assess the patients' satisfaction and quality of life (QoL) after surgery, the questionnaire of the Rustemeyer's study and Oral Health Impact Profile (OHIP) questionnaire were used, respectively. The paired-sample t-test, Spearman correlation analysis and Pearson correlation analysis was used in SPSS statistical software.

Results: In class III malocclusion patients, decrease in facial angle, convexity angle, mentolabial angle, and increase in upper lip protrusion had the most positive impact on QoL. Decrease in facial angle and increase in upper lip protrusion were correlated positively with satisfaction questionnaires. In class II malocclusion patients, increase in convexity angle, facial angle and mentolabial angle had the most positive impact on satisfaction and QoL.

Conclusions: Consideration both subjective and objective parameters affecting on the patients' satisfaction and their quality of life is essential.

Keywords: cephalometric changes; quality of life; orthognathic surgery; patient satisfaction.

Accepted for publication: 13 December 2014

To cite this article:

Baherimoghaddam T, Oshagh M, Naseri N, Nasrbadi NI, Torkan S. Changes in Cephalometric Variables after Orthognathic Surgery and Their Relationship to Patients' Quality of Life and Satisfaction

J Oral Maxillofac Res 2014;5(4):e6

URL: http://www.ejomr.org/JOMR/archives/2014/4/e6/v5n4e6ht.pdf

doi: $10.5037 /$ jomr.2014.5406
\end{abstract}




\section{INTRODUCTION}

Skeletal and dentofacial discrepancies may adversely influence the quality of life (QoL) of patients through affecting their facial appearance, oral function or both [1-3]. Patients with malocclusion, especially those severe cases that need surgical correction, are proved to have lower oral health-related quality of life (OHRQoL) [2-4]. Apart from problems with mastication and facial aesthetic, which are usually patients' chief complaint []ㅡ, they come across other difficulties such as social interactions, interpersonal relationships, and even selection of partner and profession, that all affect their OHRQoL [ $\underline{6}]$.

Nowadays, orthognathic surgeries are commonly used for patients with severe skeletal discrepancies $[\underline{7}, \underline{8}]$. When planning an orthognathic surgery, improving the patients' psychological health and correcting the patients' ability to accept their appearance are as important as their aesthetic and functional improvement $[\underline{9}, \underline{10}]$. Much attention has been paid to the factors influencing postsurgical satisfaction and subsequently improving QoL during the last decade [3, 10-14].

Despite equipment improvements and technical progress that have made the orthognathic surgeries much faster and simpler than they used to be, patients' dissatisfaction with the outcomes is still a common issue [15]. However, achieving the patients' expectations of the orthognathic surgeries has been shown to be very complicated; as not only the surgeon's skills but also the patient-surgeon communication well affects the patients' satisfaction [14].

Even in the most successful cases, patients' preoperative expectations of orthognathic surgery and postoperative outcome could offer discrepancies if they are not well informed and clarified about the outcomes and possible postsurgical discomforts. Therefore, patients may experience physical or psychological dissatisfaction, and express such dissatisfaction through formal or informal complaints [10].

Orthodontic surgical treatments are planned and conducted based on objective criteria and normative values, these may differ from patients' perception of improvement and QoL which are affected by subjective parameters [16]. Determining and considering affective soft and hard tissue parameters on patients' satisfaction and QoL must be performed before treatment planning. There is a lack of information regarding the relationship of QoL and postsurgical level of satisfaction with cephalometric changes following surgery $[\underline{3}, \underline{17}]$.
Several questionnaires have been developed in order to assess the impact of dental conditions on the $\mathrm{QoL}$ [14]. 49-item Oral Health Impact Factor (OHIP) is one of the mostly used questionnaires which could capture individuals' perceptions of the social impact of oral disorders on their well-being. The OHIP-14 questionnaire was developed by Slade as a shorter version of the OHIP-49 which focuses on oral health- specific aspects of functional limitation and physical disability, psychological, social aspects of disability, and handicaps. The OHIP-14 evaluate negative impacts of oral disorder whereas some other instruments evaluate both positive and negative impacts. However, the OHIP is the one of the bestdocumented instrument and most widely used questionnaire.

Hence, the aim of this study was to assess relationships between oral health-related quality of life and satisfaction with cephalometric changes in hard and soft tissues variables. The results of this study may help clinicians to improve patients' quality of life and satisfaction following orthognathic surgery by considering effective soft and hard tissue variables. The research hypothesis was that there are soft and hard tissue variables which are more effective in patients' level of satisfaction and quality of life.

\section{MATERIAL AND METHODS Patients}

A cohort study of orthodontic patients whose treatment plan included an orthognathic surgery was designed. Ethical permission was obtained from the ethical committee of Shiraz University of Medical Sciences. All patients who were referred to maxillofacial departments of any private or public hospitals of the city of Shiraz for orthognathic surgery by their orthodontists during March 2010 - July 2011 were invited into the study. The 25 class II and 30 class III malocclusion patients who met the specific criteria were recruited. The inclusion criteria were: to have received presurgical orthodontic treatment with the same protocol (0.022 inch standard edge wise bracket), were scheduled for a combination of a standard one-piece Le Fort I osteotomy and bilateral sagittal split ramus osteotomy (BSSRO) with rigid fixation, condylar positioning devices were not used, inter-occlusal splint were applied for two weeks. For all patients, pre- and postsurgical cephalograms were available, and all patients filled the questionnaires. Those who did not go under surgery for any reason (e.g. financial or general health issues), and who had cleft lip/palate, craniofacial syndrome, 
posttraumatic deformity, tempromandibular diseases, facial asymmetry, or mandibular border asymmetry were not recruited for the study. Moreover, among recruited ones, those who had a postoperative infection, mal-union with poor occlusion, or nerve injury, and those who refused to fill the questionnaires in full were excluded from final analysis.

\section{Lateral cephalometry}

Pre- and postsurgical cephalograms were taken with head in natural position, teeth in centric occlusion, and lips in response. The distance between film and $\mathrm{X}$-ray tube was $150 \mathrm{~cm}$ and the distance between the film and mid sagittal plane of the patient's head was $18 \mathrm{~cm}$. The horizontal reference line used in this study was the line with 7 degrees of difference to the sella-nasion line. Soft and hard tissue landmarks were shown in Figure 1. Seven angular (SNA, SNB, ANB, nasolabial angle, facial angle, convexity angle, mentolabial angle), 5 linear (upper lip length, lower lip length, upper lip protrusion, lower lip protrusion,
Wits appraisal) and one ratio-related (Jaraback index) measurements were used to assess dento-skeletal characteristics on pre- and postsurgical cephalograms of each patient (Figure 2). Pre- and postsurgical radiographs used in this study were taken when no orthodontic appliances were present on the teeth in order to avoid misinterpretations resulting from presence of brackets, especially in lips.

\section{Questionnaires}

The Persian version of the short form (14 itemed) of the Oral Health Impact Profile questionnaire (OHIP-14) [18] was used to evaluate the QoL of participants, OHIP-14 has seven domains (and two items per domain): functional limitation, physical pain, psychological discomfort, physical disability, psychological disability, social disability, and handicap. The response to each item was scored on a 5 -point scale as never (0) to very often (4). Therefore, a higher score indicated poorer QoL.

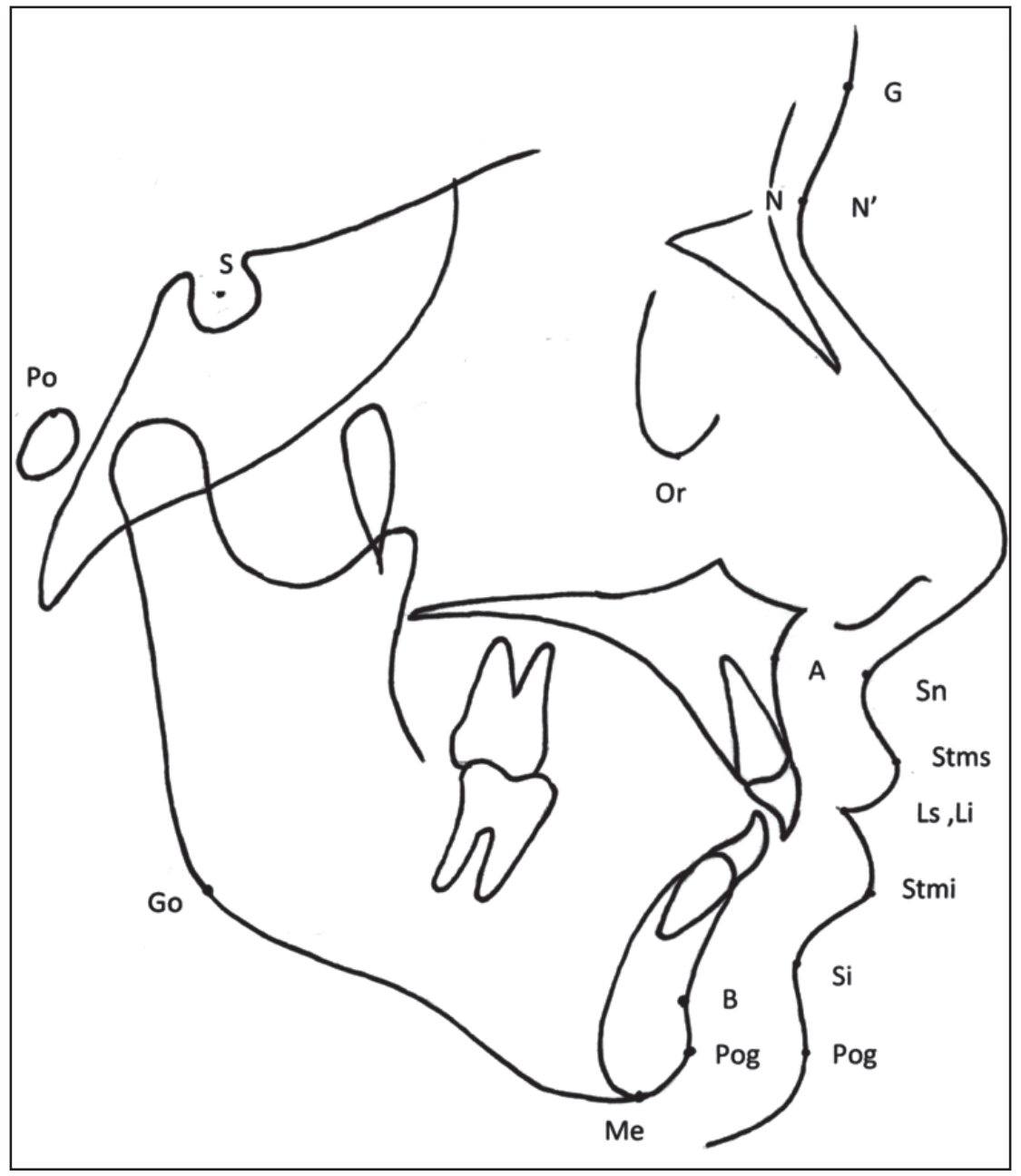

Figure 1. Soft and hard tissue cephalometric landmarks used in the study.

$\mathrm{S}=$ sella; $\mathrm{A}=$ point $\mathrm{A} ; \mathrm{B}=$ point $\mathrm{B} ; \mathrm{N}=$ nasion; $\mathrm{Go}=$ gonion; $\mathrm{Me}=$ menton; $\mathrm{Po}=$ pogonion; $\mathrm{G}=$ glabella; $\mathrm{SN}=$ subnasale; Ls = labralesuperius; $\mathrm{Li}=$ labraleinferius; $\mathrm{Stms}=$ stomionsuperius; $\mathrm{Stmi}=$ stomioninferius; $\mathrm{Pg}$ ' $=$ soft tissue pogonion; $\mathrm{Si}=$ labiomental sulcus; N'= soft tissue nasion. 


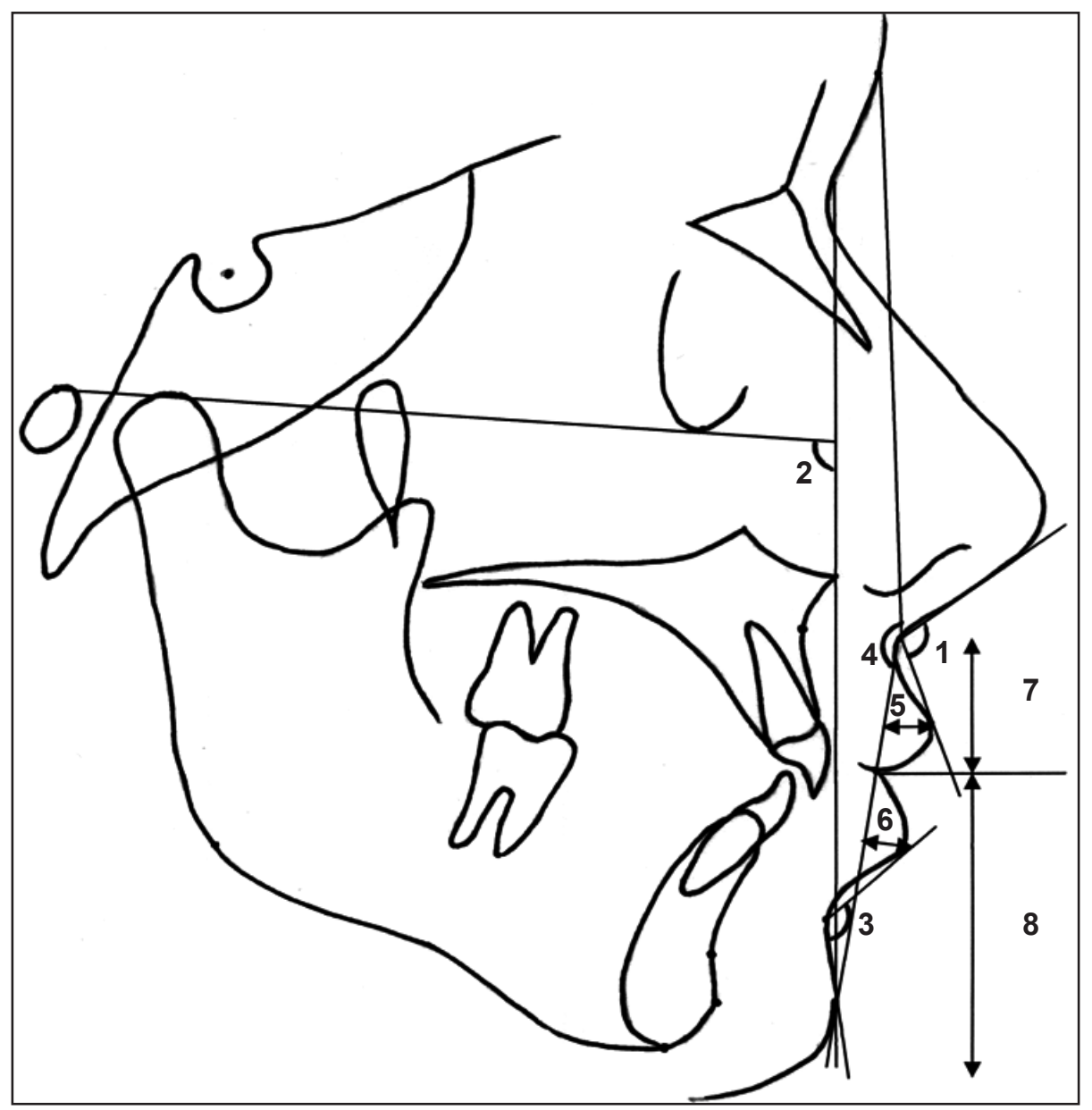

Figure 2. Soft and hard tissue measurement: $1=$ Nasolabial angle; $2=$ Facial angle; $3=$ Mentolabial angle; $4=$ Convexity angle; $5=$ Upper lip protrusion; 6 = Lower lip protrusion; 7 = Upper lip length; 8 = Lower lip length.

A short six-item form of the Rustemeyer's questionnaire [ $\underline{3}$ ] was also used to assess the participants' overall satisfaction, the relatives' and friends' opinions about the results of surgery, and aesthetic and masticatory improvements compared to before surgery via an 11-point scale based on a visual analog scale (VAS; $0=$ poor, $10=$ excellent) (Table 1). As a Persian version of the Rustemeyer's questionnaire was not available, the shortened sixitem form was translated into Persian through a forward-backward practice. Its content validity was approved by two experienced Persian speaking orthodontists, and its reliability was checked through a pilot study of 20 patients who had previously gone under orthognathic surgery. These questionnaires were answered by the patients an average 1.1 years (standard deviation 0.7 years) after surgery, in order to minimize the impression of the postsurgical complications (oedema, swelling and pain) on their responses.

Patients' socio-demographic information was obtained when consented to participate. Participants were then guided through the process of the study. They were provided with the researchers' phone number and were encouraged to ask any question they had at any point until the end of study.

Table 1. Rustemeyer's questionnaire about patients' satisfaction after surgery

\begin{tabular}{l|l}
\hline \multicolumn{1}{c|}{ Questions } & \\
\hline Please mark one grade of the scale from 0 (poor) to 10 (excellent). & \\
\hline 1. How would you assess your facial aesthetics before surgery? & $0-1-2-3-4-5-6-7-8-9-10$ \\
\hline 2. How would you assess your facial aesthetics after surgery? & $0-1-2-3-4-5-6-7-8-9-10$ \\
\hline 3. How would you assess your chewing function before surgery? & $0-1-2-3-4-5-6-7-8-9-10$ \\
\hline 4. How would you assess your chewing function today? & $0-1-2-3-4-5-6-7-8-9-10$ \\
\hline 5. How do you feel exactly about the surgical outcome of your operation? & $0-1-2-3-4-5-6-7-8-9-10$ \\
\hline 6. How do your relatives and friends feel in total about the surgical outcome of your operation? & $0-1-2-3-4-5-6-7-8-9-10$ \\
\hline
\end{tabular}




\section{Statistical analysis}

SPSS statistical software (version 18.0) was used for data manipulation. Kolmogorov-Smirnov normality test was used to determine whether the quantitative variables were normally distributed. Paired t-test was used to evaluate the difference of $\mathrm{QoL} /$ satisfaction outcomes in sexes and educational level.

Paired t-tests were used to assess changes before and after surgery. Pearson correlation analysis was performed to determine the degree of correlation between QoL and satisfaction scores with chephalometric changes. The reproducibility of the measurements was determined by randomly selecting 15 cephalograms and repeating the tracing by the same examiner, one month after the initial tracing. No significant difference was found when the t-test was performed to evaluate the repeated measurements. The intra examiner correlation (Pearson correlation) of obtained data was 0.82 .

The results and data were expressed as means and standard deviations (M [SD]).

\section{RESULTS}

The study took three and a half years to complete. Data from 30 class III (22.77 [3.78] years, 11 females and 19 males) and 25 class II malocclusion patients (25.12 [4.47] years, 14 females and 11 males) were used in the final analysis.

No significant relationship was found between OHRQoL with gender $(\mathrm{P}=0.743)$ or educational levels $(\mathrm{P}=0.534)$. Therefore, gender and educational level were not considered further. Patients' responses to questions which evaluate their information about treatment are shown in Table 2.

\section{Changes in cephalometric variables after surgery in class III malocclusion patients}

A significant decrease was detected post operatively in the soft tissue parameters: nasolabial angle $(\mathrm{P}=0.038)$, facial angle $(\mathrm{P} \leq 0.001)$, convexity angle $(\mathrm{P} \leq 0.001)$, mentolabial angle $(\mathrm{P} \leq 0.001)$, and lower lip protrusion $(\mathrm{P} \leq 0.001)$; in the hard tissue parameters: SNB $(\mathrm{P}=0.002)$. A significant increase was found in the soft tissue parameters: upper lip protrusion $(\mathrm{P} \leq 0.001)$, upper lip length $(\mathrm{P} \leq 0.003)$; in the hard tissue parameters: Jaraback index $(\mathrm{P}=0.028)$, ANB angle $(\mathrm{P} \leq 0.001)$, SNA angle $(\mathrm{P} \leq 0.003)$ and Wits appraisal $(\mathrm{P} \leq 0.001)$. On the other hand, no significant change was found in lower lip length $(\mathrm{P}=0.712)$ (Table 3$)$.

\section{Changes in cephalometric variables after surgery in class II malocclusion patients}

A significant increase was shown post operatively in the soft tissue parameters: Facial angle $(\mathrm{P} \leq 0.001)$, convexity angle $(\mathrm{P} \leq 0.001)$, and mentolabial angle $(\mathrm{P} \leq 0.001)$; in the hard tissue parameters: SNB $(\mathrm{P} \leq 0.001)$. A significant decrease was found in the soft tissue parameters: upper lip protrusion $(P \leq 0.001)$, upper lip length $(P \leq 0.001)$, lower lip protrusion $(\mathrm{P} \leq 0.004)$, and lower lip length $(\mathrm{P} \leq 0.004)$; in the hard tissue parameters: ANB $(\mathrm{P} \leq 0.001)$, SNA $(\mathrm{P}=0.008)$ and Wits appraisal $(\mathrm{P} \leq 0.001)$. Nevertheless, no significant change was found in Jaraback index $(\mathrm{P}=0.578)$, nasolabial angle $(\mathrm{P}=0.559)$ (Table 3$)$.

\section{Correlations between changes in cephalometric variables and questionnaires items}

Several cephalometric variables were significantly correlated with OHRQoL and satisfaction levels in class III malocclusion patients. Facial angle, convexity angle, mentolabial angle, nasolabial angle, and upper lip protrusion had the most significant correlations with questionnaires items. Reduction in mentolabial angle, facial angle, and convexity angle was correlated positively with functional limitation (OH-2), psychological discomfort (OH-5; OH-6), psychological disability (OH-9), and handicap (facial angle with $\mathrm{OH}-13$ and $\mathrm{OH}-14$, mentolabial angle just with $\mathrm{OH}-13$, and convexity angle just with $\mathrm{OH}-14$ ). In contrast, reduction in nasolabial angle and increase in upper lip protrusion were correlated negatively with functional limitation (OH-1), psychological discomfort (OH-5; OH-6), psychological disability (OH-9), and handicap (OH-13, OH-14). The changes in Wits appraisal and ANB angle revealed positive correlation with changes in scores of $\mathrm{OH}-3$ (functional limitation) and $\mathrm{OH}-4$ (physical pain) (Table 4).

Table 2. Patients' information about treatment

\begin{tabular}{|c|c|c|c|}
\hline Questions & Sufficiently & Reasonably & Far too little \\
\hline Were the difference treatment relatives explained to you clearly before surgery? & 52 & 2 & 1 \\
\hline Were you given adequate information about different procedure before surgery? & 47 & 5 & 3 \\
\hline Did you talk about your expectations and motivation with your surgeon? & 45 & 9 & 1 \\
\hline
\end{tabular}


Table 3. Cephalometric changes before and $1.6(0.7)$ years after surgery

\begin{tabular}{|c|c|c|c|c|}
\hline \multirow[b]{2}{*}{ Parameters } & \multirow{2}{*}{$\begin{array}{c}\text { Type of } \\
\text { deformity }\end{array}$} & Presurgery & Postsurgery & \multirow[b]{2}{*}{$P$ value } \\
\hline & & $\begin{array}{c}\text { Median } \\
\text { Mean (SD) }\end{array}$ & $\begin{array}{c}\text { Median } \\
\text { Mean (SD) }\end{array}$ & \\
\hline \multirow{2}{*}{ SNA } & $\mathrm{Cl}$ III & $82.4(5.14)$ & $85.18(3.86)$ & $0.003^{\mathrm{a}}$ \\
\hline & $\mathrm{Cl}$ II & $83.7(5.38)$ & $85.9(7.88)$ & $0.008^{\mathrm{a}}$ \\
\hline \multirow{2}{*}{ SNB } & $\mathrm{Cl}$ III & $84.63(4.68)$ & $81.98(3.64)$ & $0.002^{\mathrm{a}}$ \\
\hline & $\mathrm{Cl}$ II & $75.4(5.13)$ & $81.5(7.93)$ & $<0.001^{\mathrm{b}}$ \\
\hline \multirow{2}{*}{ ANB } & $\mathrm{Cl}$ III & $-0.57(3.7)$ & $2.47(2.15)$ & $<0.001^{\mathrm{b}}$ \\
\hline & $\mathrm{Cl}$ II & $8.3(4.08)$ & $4.4(2.7)$ & $<0.001^{\mathrm{b}}$ \\
\hline \multirow{2}{*}{ Wits appraisal } & $\mathrm{Cl}$ III & $-7.07(4.58)$ & $-0.39(3.06)$ & $<0.001^{\mathrm{b}}$ \\
\hline & $\mathrm{Cl}$ II & $6.12(3.84)$ & $2.88(2.84)$ & $<0.001^{\mathrm{b}}$ \\
\hline \multirow{2}{*}{ Jaraback index } & $\mathrm{Cl}$ III & $61.23(10.62)$ & $64.87(7.99)$ & $0.028^{\mathrm{a}}$ \\
\hline & $\mathrm{Cl} \mathrm{II}$ & $63.36(8.7)$ & $62.76(6.41)$ & 0.578 \\
\hline \multirow{2}{*}{ Upper lip length } & $\mathrm{Cl}$ III & $21.95(3.3)$ & $23.3(3.04)$ & $0.003^{\mathrm{a}}$ \\
\hline & $\mathrm{Cl}$ II & $24.52(3.56)$ & $22.58(3.92)$ & $0.001^{\mathrm{a}}$ \\
\hline \multirow{2}{*}{ Lower lip length } & $\mathrm{Cl}$ III & $54.43(7.03)$ & $54.03(5.87)$ & 0.712 \\
\hline & $\mathrm{Cl} \mathrm{II}$ & $48.56(5.77)$ & $45.64(5.17)$ & 0.004 \\
\hline \multirow{2}{*}{ Nasolabial angle } & $\mathrm{Cl}$ III & $92.13(4.45)$ & $90.07(5.54)$ & $0.038^{\mathrm{a}}$ \\
\hline & $\mathrm{Cl}$ II & $110.64(10.93)$ & $109.16(11.44)$ & 0.559 \\
\hline \multirow{2}{*}{ Facial angle } & $\mathrm{Cl}$ III & $92.3(3.4)$ & $87.27(3.85)$ & $<0.001^{\mathrm{b}}$ \\
\hline & $\mathrm{Cl}$ II & $85.56(6.73)$ & $90.2(4.97)$ & $<0.001^{\mathrm{b}}$ \\
\hline \multirow{2}{*}{ Convexity angle } & Cl III & $176.37(3.79)$ & $170.5(3.05)$ & $<0.001^{\mathrm{b}}$ \\
\hline & $\mathrm{Cl}$ II & $162.08(5.07)$ & $167.52(4.62)$ & $<0.001^{\mathrm{b}}$ \\
\hline \multirow{2}{*}{ Mentolabial angle } & $\mathrm{Cl}$ III & $138.26(13.02)$ & $120.03(15.16)$ & $<0.001^{\mathrm{b}}$ \\
\hline & $\mathrm{Cl}$ II & $117.64(18.98)$ & $122.8(17.19)$ & $<0.001^{\mathrm{b}}$ \\
\hline \multirow{2}{*}{ Upper lip protrusion } & $\mathrm{Cl}$ III & $2.45(1.57)$ & $4.98(2.27)$ & $<0.001^{\mathrm{b}}$ \\
\hline & $\mathrm{Cl}$ II & $7(2.3)$ & $5.26(2.27)$ & $<0.001^{\mathrm{b}}$ \\
\hline \multirow{2}{*}{ Lower lip protrusion } & $\mathrm{Cl}$ III & $5.75(2.42)$ & $3.87(2.78)$ & $<0.001^{\mathrm{b}}$ \\
\hline & $\mathrm{Cl}$ II & $5.12(2.29)$ & $3.88(3.48)$ & $0.004^{\mathrm{a}}$ \\
\hline
\end{tabular}

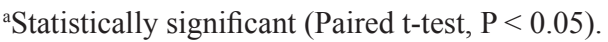

${ }^{b}$ Statistically highly significant (Paired t-test, $\mathrm{P}<0.001$ ).

$\mathrm{SD}=$ standard deviation

Table 4. Correlations between changes in cephalometric variables and Oral Health Impact Profile questionnaire (OHIP) items in class III malocclusion patients

\begin{tabular}{|c|c|c|c|c|c|c|c|c|c|c|c|c|}
\hline Parameters & OH-1 & OH-3 & OH-4 & OH-5 & OH-6 & OH-7 & OH-9 & OH-10 & OH-11 & OH-12 & OH-13 & OH-14 \\
\hline SNA & n.s & $0.442^{\mathrm{a}}$ & n.s & n.s & n.s & n.s & n.s & n.s & n.s & n.s & n.s & n.s \\
\hline SNB & n.s & n.s & n.s & n.s & n.s & n.s & n.s & n.s & n.s & n.s & n.s & n.s \\
\hline ANB & n.s & $0.475^{\mathrm{a}}$ & n.s & n.s & n.s & n.s & n.s & $-0.429^{a}$ & $-0.465^{b}$ & n.s & n.s & n.s \\
\hline Wits appraisal & n.s & $0.51^{\mathrm{a}}$ & $-0.537^{\mathrm{b}}$ & n.s & n.s & $-0.403^{\mathrm{a}}$ & n.s & n.s & $-0.503^{b}$ & n.s & n.s & n.s \\
\hline Upper lip length & n.s & n.s & n.s & n.s & $0.364^{\mathrm{a}}$ & n.s & n.s & n.s & n.s & n.s & n.s & n.s \\
\hline Lower lip length & n.s & n.s & $0.372^{\mathrm{a}}$ & n.s & n.s & n.s & n.s & n.s & n.s & n.s & n.s & n.s \\
\hline Nasolabial angle & $-0.417^{\mathrm{a}}$ & n.s & n.s & $-0.43^{\mathrm{a}}$ & $-0.472^{\mathrm{a}}$ & n.s & $-0.412^{\mathrm{a}}$ & n.s & n.s & n.s & n.s & n.s \\
\hline Facial angle & $0.556^{\mathrm{b}}$ & n.s & n.s & $0.599^{\mathrm{b}}$ & $0.499^{\mathrm{b}}$ & n.s & $0.517^{\mathrm{b}}$ & n.s & n.s & $0.489^{\mathrm{b}}$ & $0.545^{\mathrm{b}}$ & $0.424^{\mathrm{a}}$ \\
\hline Convexity angle & $0.464^{\mathrm{b}}$ & n.s & n.s & $0.499^{\mathrm{b}}$ & $0.425^{\mathrm{a}}$ & n.s & $0.381^{\mathrm{a}}$ & n.s & n.s & n.s & n.s & $0.37^{\mathrm{a}}$ \\
\hline Mentolabial angle & $0.447^{\mathrm{a}}$ & n.s & n.s & $0.5^{\mathrm{b}}$ & $0.429^{\mathrm{a}}$ & n.s & $0.541^{\mathrm{a}}$ & n.s & n.s & n.s & $0.382^{\mathrm{a}}$ & n.s \\
\hline Upper lip protrusion & $-0.529^{b}$ & n.s & n.s & $-0.656^{b}$ & $-0.567^{\mathrm{b}}$ & n.s & $-0.44^{\mathrm{a}}$ & n.s & n.s & n.s & $-0.535^{b}$ & $-0.433^{\mathrm{a}}$ \\
\hline Lower lip protrusion & n.s & n.s & n.s & n.s & n.s & n.s & n.s & n.s & n.s & n.s & n.s & n.s \\
\hline Jaraback index & n.s & n.s & n.s & n.s & n.s & n.s & n.s & n.s & n.s & n.s & n.s & n.s \\
\hline
\end{tabular}

${ }^{a}$ Statistically significant (Pearson correlations, $\mathrm{P}<0.05$ ).

${ }^{b}$ Statistically highly significant (Pearson correlations, $\mathrm{P}<0.001$ ).

$\mathrm{n} . \mathrm{s}=$ Statistically non significant (Pearson correlations, $\mathrm{P}>0.05)$ 
Table 5. Correlations between cephalometric changes and Oral Health Impact Profile questionnaire (OHIP) items in class II malocclusion patients

\begin{tabular}{|c|c|c|c|c|c|c|c|c|c|c|}
\hline Parameters & OH-3 & OH-4 & OH-5 & OH-6 & OH-7 & OH-9 & OH-10 & OH-11 & OH-13 & OH-14 \\
\hline SNA & n.s & n.s & n.s & n.s & n.s & n.s & n.s & n.s & n.s & n.s \\
\hline SNB & n.s & n.s & n.s & n.s & n.s & n.s & n.s & n.s & n.s & n.s \\
\hline ANB & n.s & n.s & n.s & n.s & $0.488^{\mathrm{a}}$ & n.s & $0.67^{\mathrm{b}}$ & $0.678^{\mathrm{b}}$ & n.s & n.s \\
\hline Wits appraisal & $-0.517^{b}$ & n.s & $0.417^{\mathrm{a}}$ & n.s & $0.636^{\mathrm{b}}$ & n.s & n.s & n.s & $0.539^{\mathrm{b}}$ & n.s \\
\hline Upper lip length & n.s & n.s & n.s & n.s & n.s & n.s & $-0.438^{a}$ & $-0.4^{\mathrm{a}}$ & n.s & n.s \\
\hline Lower lip length & n.s & n.s & n.s & n.s & n.s & n.s & $-0.456^{a}$ & $-0.407^{\mathrm{a}}$ & n.s & n.s \\
\hline Nasolabial angle & n.s & $-0.429^{a}$ & n.s & n.s & n.s & n.s & n.s & n.s & n.s & n.s \\
\hline Facial angle & n.s & n.s & $-0.453^{\mathrm{a}}$ & $-0.446^{\mathrm{a}}$ & n.s & n.s & n.s & n.s & $-0.508^{\mathrm{a}}$ & $-0.415^{a}$ \\
\hline Convexity angle & n.s & n.s & $-0.578^{b}$ & n.s & $0^{\mathrm{a}}$ & $-0.599^{b}$ & $-0.461^{\mathrm{a}}$ & $-0.45^{\mathrm{a}}$ & $-0.678^{b}$ & $-0.581^{b}$ \\
\hline Mentolabial angle & n.s & n.s & $-0.448^{a}$ & $-0.479^{a}$ & $-0.507^{b}$ & $-0.531^{b}$ & n.s & n.s & $-0.695^{b}$ & $-0.574^{b}$ \\
\hline Upper lip protrusion & n.s & n.s & n.s & n.s & n.s & n.s & n.s & n.s & n.s & n.s \\
\hline Lower lip protrusion & n.s & n.s & n.s & n.s & n.s & n.s & n.s & n.s & $0.645^{b}$ & $0.525^{\mathrm{b}}$ \\
\hline Jaraback index & n.s & n.s & n.s & n.s & n.s & n.s & n.s & n.s & n.s & n.s \\
\hline
\end{tabular}

aStatistically significant (Pearson correlations, $\mathrm{P}<0.05$ ).

${ }^{b}$ Statistically highly significant (Pearson correlations, $\mathrm{P}<0.001$ ).

$\mathrm{n} . \mathrm{s}=$ Statistically non significant $($ Pearson correlations, $\mathrm{P}>0.05)$

Also, upper lip protrusion, facial angle, mentolabial angle, and nasolabial angle was significantly correlated with overall satisfaction (Q5) (Table 6).

In class II malocclusion patients, convexity angle, facial angle, lower lip protrusion, and mentolabial angle were correlated negatively with the changes in OHIP-14 scores involving psychological discomfort $(\mathrm{OH}-5)$, physical disability (OH-7), psychological disability (OH-9), and handicaps (OH-13; OH-14). The changes in Wits appraisal revealed positive correlation with $\mathrm{OH}-3$ (functional limitation) (Table 5). Changes in mentolabial angle, facial angle, and convexity angle were significantly correlated with overall satisfaction (Q5), relatives' opinions (Q6), and the difference between facial aesthetic before and after surgery (Q2-Q1) (Table 6).

\section{DISCUSSION}

Patient centred evaluation has been valuable in defining patients' perception and expectation of treatment. It can provide an overall picture of patients' expectation which determine treatment effectiveness [11]. There is a close relationship between satisfaction and psychosocial functioning [12]. Satisfactory results of an orthognathic surgery from clinician's point of view may not be as satisfactory from patient's aspect [19].

Table 6. Correlations between cephalometric changes and satisfaction questions in class III and II malocclusion patients

\begin{tabular}{|c|c|c|c|c|c|c|c|c|}
\hline \multirow{2}{*}{ Parameters } & \multicolumn{4}{|c|}{ Class III } & \multicolumn{4}{|c|}{ Class II } \\
\hline & Q 2-1 ${ }^{\mathrm{a}}$ & Q 4-3 ${ }^{\mathrm{a}}$ & Q5 & Q6 & Q 2-1 & Q 4-3 & Q5 & Q6 \\
\hline SNA & n.s & n.s & n.s & n.s & n.s & n.s & n.s & n.s \\
\hline SNB & n.s & n.s & n.s & n.s & n.s & n.s & n.s & n.s \\
\hline ANB & n.s & n.s & n.s & & n.s & n.s & n.s & $-0.58^{\mathrm{b}}$ \\
\hline Wits appraisal & n.s & n.s & n.s & $0.458^{\mathrm{a}}$ & $-0.445^{\mathrm{a}}$ & $-0.449^{a}$ & $-0.49^{a}$ & $-0.668^{b}$ \\
\hline Upper lip length & n.s & n.s & n.s & n.s & n.s & n.s & n.s & n.s \\
\hline Lower lip length & n.s & n.s & n.s & n.s & n.s & n.s & n.s & n.s \\
\hline Nasolabial angle & n.s & n.s & $0.398^{\mathrm{a}}$ & n.s & n.s & n.s & n.s & n.s \\
\hline Facial angle & n.s & $-0.431^{\mathrm{a}}$ & $-0.697^{\mathrm{b}}$ & n.s & $0.563^{\mathrm{b}}$ & $0.604^{\mathrm{b}}$ & $0.928^{\mathrm{b}}$ & n.s \\
\hline Convexity angle & n.s & n.s & n.s & n.s & $0.478^{\mathrm{a}}$ & $0.498^{\mathrm{a}}$ & $0.846^{\mathrm{b}}$ & n.s \\
\hline Mentolabial angle & n.s & n.s & $-0.426^{\mathrm{a}}$ & n.s & $0.521^{\mathrm{b}}$ & $0.559^{\mathrm{b}}$ & $0.844^{\mathrm{b}}$ & n.s \\
\hline Upper lip protrusion & n.s & n.s & $0.488^{\mathrm{b}}$ & n.s & n.s & n.s & n.s & n.s \\
\hline Lower lip protrusion & n.s & n.s & n.s & n.s & $0.498^{\mathrm{b}}$ & $0.551^{\mathrm{b}}$ & n.s & n.s \\
\hline Jaraback index & n.s & n.s & n.s & n.s & n.s & n.s & n.s & n.s \\
\hline
\end{tabular}

aStatistically significant (Pearson correlations, $\mathrm{P}<0.05$ ).

${ }^{\mathrm{b} S t a t i s t i c a l l y ~ h i g h l y ~ s i g n i f i c a n t ~(P e a r s o n ~ c o r r e l a t i o n s, ~} \mathrm{P}<0.001$ ).

$\mathrm{n} . \mathrm{s}=$ Statistically non significant (Pearson correlations, P > 0.05); Q 2-1 = difference between Q2 and Q1; Q 4-3 = difference between Q4 and Q3. 
Facial disproportionate, in contrast with facial conformity (symmetry), is unattractive, and unacceptable [20].

Differences between predicted and postsurgical outcomes could be measured with a range of $1-2^{\circ}$ for SNA, SNB, and ArGoMe in cases of bimaxillary osteotomy, cephalometric prediction remains an accurate tool for treatment planning [21]. But, treatments that are merely based on cephalometric evaluation lead to inadequate correction of facial beauty and therefore, patient dissatisfaction [22].

Various factors may affect postsurgical satisfaction in a positive direction [11-13]. Patient's awareness about the procedures of orthodontic surgical treatment can improves patient's level of satisfaction [14]. In the present study, as previously indicated, most of the patients have received adequate information about the treatment procedures, thus the effect of inadequate information on the reduction of the level of satisfaction and QoL is negligible.

In the present study, patients with postoperative infections, mal-union with poor occlusions and nerve injury were excluded. It has been shown that patients who experienced unexpected problems such as of postoperative infections, mal-union with poor occlusions and nerve injury following surgery were more likely to be dissatisfied with the treatment [11]. A negative tendency on patients rating could be observed if postoperative problems were persisted [3] . Therefore we tried to reduce other affective factors on patients' satisfaction and QoL to clarify the effect of cephalometric variables changes on patients' satisfaction and QoL and eliminate differences between groups.

No significant differences was observed in the result obtained from all approaches between two genders, this finding is consistent with those studies which did not find any association between gender and QoL outcomes [프, 23, 24]. However, Nicodemo et al. [25] reported that women showed improved selfesteem and diminished depressive symptom 6 months after orthognathic surgery, whereas men showed no improvement.

As in many studies in this field, we observed significant changes in the hard and soft tissue parameters after orthodontic surgical treatment. However, lower lip length in class III and nasolabial angle and Jaraback index in class II malocclusion showed no significant changes after surgery. Chew et al. [26] showed no significant changes in mentolabial fold thickness (mm) after orthognathic surgery in class III malocclusion patients; in the present study changes in mentolabial area was assessed by mantolabial angle instead of mentolabial fold thickness. In other hand,
Rustemeyer et al. [27] showed significant changes in mentolabial angle only in class II malocclusion patients following orthognathic surgery. This disagreement could be related to the high method error and variability in this area, which by reported by Fernandez-Riveiro et al. [28].

Findings from this study showed that some objective changes of cephalometric variable are effective in improving the patients' QoL and satisfaction. Correction of sagittal aspect of deformity by improving facial angle and convexity angle compared with Jaraback index which used to determine changes in vertical aspect of deformity, played an important role in increasing QoL and patients' satisfaction in both groups. This is in great agreement with other studies which have shown that the most important affecting factor on facial attractiveness is the anteroposterior dimension [29, $\underline{30}]$.

A number of studies have shown that increase in lower facial height is more acceptable while other studies claim the opposite $[\underline{31}, \underline{32}]$. In the current study, although pre- and postsurgical lower facial height had a significant difference in class III malocclusion patients, no significant correlation were observed between changes in lower facial height and QoL and satisfaction levels. It has been indicated that alteration of the appearance was related more to the sagittal SNB angle than vertical ArGoMe angle, no correlation between vertical ArGoMe angle and level of patient satisfaction was reported in class III malocclusion patients []ㅡ.

As reported by Rustemeyer et al. [17], mentolabial angle and nasion-pogonion have positive correlation with QoL and postsurgical satisfaction in class III malocclusion patients. Unlike the present study, they found that the reduction in SNB, facial convexity angle, and lower lip protrusion has negative effect on QoL. This disagreement could be due to the shorter follow-up period of their study which was carried out only 8 months after surgery, while the mean range of follow-up period in the present study was $1.1(0.7)$ years. It has been shown that in one third of patients, it takes 24 months to get used to their new facial appearance [33].

Present study showed that increasing the upper lip prominence had significant impact on QoL of class III malocclusion patients. Also, changes in lower lip prominence, in class II malocclusion patients, was significantly correlated with patients' satisfaction and QoL. Chew et al. [34] reported that lower lip prominence was the only cephalometric variable that significantly influenced clinicians' evaluation of profile aesthetics; although, lay persons found no difference between lower lip and upper lip attractiveness. 
As a good increase in the QoL is expected from orthognathic surgeries [1, 2$]$, attention to the parameters affecting the patients' satisfaction and their QoL is essential, particularly in treatment planning. Enhancing patients' awareness about facial aesthetics which can improve facial appearance, as well as increasing patients' information regarding surgery may be useful. In this study, the hard and soft tissue changes in cephalograms were evaluated. But it is recommended that changes in profile and frontal views of the face could be evaluated in future studies. Results of this study could be beneficial for determining affective factors in the improving of QoL and patients' satisfaction which can determine treatment effectiveness. Emphasis on specific variables during treatment planning could improve patients' QoL more.

\section{CONSLUSIONS}

- In class III malocclusion patients, changes in mentolabial angle, convexity angle, facial angle, and upper lip protrusion were significantly correlated with improvements in patient' satisfaction and QoL.

- In class II malocclusion patients, changes in facial angle, convexity angle, lower lip protrusion, and mentolabial angle were significantly correlated with improvements in patient' satisfaction and QoL.

- Although some variables had significant correlation with patients' satisfaction and quality of life, it seemed that the associations were moderate.

- It is undeniable that considering the subjective and objective parameters during preparing the patients to their new appearance are necessary.

\section{ACKNOWLEDGMENTS AND DISCLOSURE STATEMENTS}

The authors report no conflicts of interest related to this study.

\section{REFERENCES}

1. Esperão PT, de Oliveira BH, de Oliveira Almeida MA, Kiyak HA, Miguel JA. Oral health-related quality of life in orthognathic surgery patients. Am J Orthod Dentofacial Orthop. 2010 Jun;137(6):790-5. [Medline: 20685534] [doi: 10.1016/j.ajodo.2008.08.031]

2. Khadka A, Liu Y, Li J, Zhu S, Luo E, Feng G, Hu J. Changes in quality of life after orthognathic surgery: a comparison based on the involvement of the occlusion. Oral Surg Oral Med Oral Pathol Oral Radiol Endod. 2011 Dec;112(6):719-25. Epub 2011 Mar 31. [Medline: 21458328] [doi: 10.1016/j.tripleo.2011.01.002]

3. Rustemeyer J, Eke Z, Bremerich A. Perception of improvement after orthognathic surgery: the important variables affecting patient satisfaction. Oral Maxillofac Surg. 2010 Sep;14(3):155-62. [Medline: 20306101] [PMC free article: 2928919] [doi: 10.1007/s10006-010-0212-2]

4. Murphy C, Kearns G, Sleeman D, Cronin M, Allen PF. The clinical relevance of orthognathic surgery on quality of life. Int J Oral Maxillofac Surg. 2011 Sep;40(9):926-30. Epub 2011 May 26. [Medline: 21616638] [doi: 10.1016/j.ijom.2011.04.001]

5. Macgregor FC. Facial disfigurement: problems and management of social interaction and implications for mental health. Aesthetic Plast Surg. 1990 Fall;14(4):249-57. [Medline: 2239515] [doi: 10.1007/BF01578358]

6. Choi WS, Lee S, McGrath C, Samman N. Change in quality of life after combined orthodontic-surgical treatment of dentofacial deformities. Oral Surg Oral Med Oral Pathol Oral Radiol Endod. 2010 Jan;109(1):46-51. [Medline: 20123378] [doi: 10.1016/j.tripleo.2009.08.019]

7. Hunt OT, Johnston CD, Hepper PG, Burden DJ. The psychosocial impact of orthognathic surgery: a systematic review. Am J Orthod Dentofacial Orthop. 2001 Nov;120(5):490-7. Review. [Medline: 11709666] [doi: 10.1067/mod.2001.118402]

8. Frost V, Peterson G. Psychological aspects of orthognathic surgery: how people respond to facial change. Oral Surg Oral Med Oral Pathol. 1991 May;71(5):538-42. [Medline: 2047092] [doi: 10.1016/0030-4220(91)90357-I]

9. Hudson JW, Jaffrey BJ, Davis C, Witkowski CE. The psychological and behavioral considerations of orthognathic surgery on identical (monozygotic) twins. Oral Surg Oral Med Oral Pathol. 1989 Sep;68(3):259-63. [Medline: 2771370] [doi: 10.1016/0030-4220(89)90206-5]

10. Flanary CM, Alexander JM. Patient responses to the orthognathic surgical experience: factors leading to dissatisfaction. J Oral Maxillofac Surg. 1983 Dec;41(12):770-4. [Medline: 6581279] [doi: 10.1016/S0278-2391(83)80042-1]

11. Chen B, Zhang ZK, Wang X. Factors influencing postoperative satisfaction of orthognathic surgery patients. Int J Adult Orthodon Orthognath Surg. 2002 Fall;17(3):217-22. [Medline: 12353939]

12. Oland J, Jensen J, Elklit A, Melsen B. Motives for surgical-orthodontic treatment and effect of treatment on psychosocial well-being and satisfaction: a prospective study of 118 patients. J Oral Maxillofac Surg. 2011 Jan;69(1):104-13. Epub 2010 Nov 2. [Medline: 21050649] [doi: 10.1016/j.joms.2010.06.203] 
13. Phillips C, Kiyak HA, Bloomquist D, Turvey TA. Perceptions of recovery and satisfaction in the short term after orthognathic surgery. J Oral Maxillofac Surg. 2004 May;62(5):535-44. [Medline: 15122555] [PMC free article: 3593632 ] [doi: 10.1016/i.joms.2003.08.025]

14. Nurminen L, Pietilä T, Vinkka-Puhakka H. Motivation for and satisfaction with orthodontic-surgical treatment: a retrospective study of 28 patients. Eur J Orthod. 1999 Feb;21(1):79-87. [Medline: 10191581] [doi: 10.1093/ejo/21.1.79]

15. Kim SJ, Kim MR, Shin SW, Chun YS, Kim EJ. Evaluation on the psychosocial status of orthognathic surgery patients. Oral Surg Oral Med Oral Pathol Oral Radiol Endod. 2009 Dec;108(6):828-32. [Medline: 19913723] [doi: 10.1016/j.tripleo.2009.07.044]

16. Allen PF. Assessment of oral health related quality of life. Health Qual Life Outcomes. 2003 Sep 8;1:40. Review. [Medline: 14514355] [PMC free article: 201012] [doi: 10.1186/1477-7525-1-40]

17. Rustemeyer J, Martin A, Gregersen J. Changes in quality of life and their relation to cephalometric changes in orthognathic surgery patients. Angle Orthod. 2012 Mar;82(2):235-41. Epub 2011 Aug 29. [Medline: 21875314] [doi: $10.2319 / 042211-285.1]$

18. Ravaghi V, Farrahi-Avval N, Locker D, Underwood M. Validation of the Persian short version of the Oral Health Impact Profile (OHIP-14). Oral Health Prev Dent. 2010;8(3):229-35. [Medline: 20848000]

19. Ahmed B, Gilthorpe MS, Bedi R. Agreement between normative and perceived orthodontic need amongst deprived multiethnic school children in London. Clin Orthod Res. 2001 May;4(2):65-71. [Medline: 11553087] [doi: 10.1034/j.1600-0544.2001.040202.x]

20. Naini FB, Cobourne MT, McDonald F, Donaldson AN. The influence of craniofacial to standing height proportion on perceived attractiveness. Int J Oral Maxillofac Surg. 2008 Oct;37(10):877-85. Epub 2008 Sep 7. [Medline: 18778915] [doi: 10.1016/j.ijom.2008.07.022]

21. Rustemeyer J, Groddeck A, Zwerger S, Bremerich A. The accuracy of two-dimensional planning for routine orthognathic surgery. Br J Oral Maxillofac Surg. 2010 Jun;48(4):271-5. Epub 2009 Jul 24. [Medline: 19632014] [doi: 10.1016/j.bjoms.2009.06.018]

22. Marşan G, Cura N, Emekli U. Soft and hard tissue changes after bimaxillary surgery in Turkish female Class III patients. J Craniomaxillofac Surg. 2009 Jan;37(1):8-17. Epub 2008 Sep 10. [Medline: 18786833] [doi: 10.1016/j.jcms.2008.07.004]

23. Pahkala RH, Kellokoski JK. Surgical-orthodontic treatment and patients' functional and psychosocial well-being. Am J Orthod Dentofacial Orthop. 2007 Aug;132(2):158-64. [Medline: 17693364] [doi: 10.1016/j.ajodo.2005.09.033]

24. Finlay PM, Atkinson JM, Moos KF. Orthognathic surgery: patient expectations; psychological profile and satisfaction with outcome. Br J Oral Maxillofac Surg. 1995 Feb;33(1):9-14. [Medline: 7718535] [doi: 10.1016/0266-4356(95)90078-0]

25. Nicodemo D, Pereira MD, Ferreira LM. Effect of orthognathic surgery for class III correction on quality of life as measured by SF-36. Int J Oral Maxillofac Surg. 2008 Feb;37(2):131-4. Epub 2007 Oct 4. [Medline: 17919889] [doi: 10.1016/j.ijom.2007.07.024]

26. Chew MT. Soft and hard tissue changes after bimaxillary surgery in Chinese Class III patients. Angle Orthod. 2005 Nov;75(6):959-63. [Medline: 16448238]

27. Rustemeyer J, Martin A. Soft tissue response in orthognathic surgery patients treated by bimaxillary osteotomy: cephalometry compared with 2-D photogrammetry. Oral Maxillofac Surg. 2013 Mar;17(1):33-41. Epub 2012 May 5. [Medline: 22562282] [PMC free article: 3576550] [doi: 10.1007/s10006-012-0330-0]

28. Fernández-Riveiro P, Smyth-Chamosa E, Suárez-Quintanilla D, Suárez-Cunqueiro M. Angular photogrammetric analysis of the soft tissue facial profile. Eur J Orthod. 2003 Aug;25(4):393-9. [Medline: 12938846] [doi: 10.1093/ejo/25.4.393]

29. Phillips C, Griffin T, Bennett E. Perception of facial attractiveness by patients, peers, and professionals. Int J Adult Orthodon Orthognath Surg. 1995;10(2):127-35. [Medline: 9081998]

30. Lucker GW, Graber LW. Physiognomic features and facial appearance judgments in children. J Psychol. 1980 Mar;104(2):261-8.

31. Johnston DJ, Hunt O, Johnston CD, Burden DJ, Stevenson M, Hepper P. The influence of lower face vertical proportion on facial attractiveness. Eur J Orthod. 2005 Aug;27(4):349-54. Epub 2005 Jun 16. [Medline: 15961569] [doi: 10.1093/ejo/cji023]

32. Erbay EF, Caniklioğlu CM. Soft tissue profile in Anatolian Turkish adults: Part II. Comparison of different soft tissue analyses in the evaluation of beauty. Am J Orthod Dentofacial Orthop. 2002 Jan;121(1):65-72. [Medline: 11786874] [doi: 10.1067/mod.2002.119573]

33. Türker N, Varol A, Ogel K, Basa S. Perceptions of preoperative expectations and postoperative outcomes from orthognathic surgery: part I: Turkish female patients. Int J Oral Maxillofac Surg. 2008 Aug;37(8):710-5. Epub 2008 Jun 9. [Medline: 18539437] [doi: 10.1016/j.ijom.2008.04.014]

34. Chew MT, Sandham A, Soh J, Wong HB. Outcome of orthognathic surgery in Chinese patients. A subjective and objective evaluation. Angle Orthod. 2007 Sep;77(5):845-50. [Medline: 17685780] [doi: 10.2319/091906-378] 


\section{To cite this article:}

Baherimoghaddam T, Oshagh M, Naseri N, Nasrbadi NI, Torkan S. Changes in Cephalometric Variables after Orthognathic Surgery and Their Relationship to Patients' Quality of Life and Satisfaction.

J Oral Maxillofac Res 2014;5(4):e6

URL: http://www.ejomr.org/JOMR/archives/2014/4/e6/v5n4e6ht.pdf

doi: $10.5037 /$ jomr.2014.5406

Copyright (C) Baherimoghaddam T, Oshagh M, Naseri N, Nasrbadi NI, Torkan S. Published in the JOURNAL OF ORAL \& MAXILLOFACIAL RESEARCH (http://www.ejomr.org), 29 December 2014.

This is an open-access article, first published in the JOURNAL OF ORAL \& MAXILLOFACIAL RESEARCH, distributed under the terms of the Creative Commons Attribution-Noncommercial-No Derivative Works 3.0 Unported License, which permits unrestricted non-commercial use, distribution, and reproduction in any medium, provided the original work and is properly cited. The copyright, license information and link to the original publication on (http://www.ejomr.org) must be included. 